Research Paper:

\title{
The Effect of Body Position and Early Ambulation on crosslark Comfort, Bleeding, and Ecchymosis After Diagnostic Cardiac Catheterization
}

Vahid Naseri Salahshour ${ }^{1}$, Masoume Sabzali Gol ${ }^{2 *}$, Shiva Sadat Basaampour ${ }^{3}$, Shokouh Varaei ${ }^{3}$, Mahbobeh Sajadi ${ }^{4}$, Abbas $_{\text {Mehran }}^{5}$

1. Student Research Committee, School of Nursing and Midwifery, Arak University of Medical Sciences, Arak, Iran.

2. Department of Medical Surgical Nursing, Faculty of Nursing and Midwifery, Karaj Branch, Islamic Azad University, Karaj, Iran.

3. Department of Medical Surgical Nursing, School of Nursing and Midwifery, Tehran University of Medical Sciences, Tehran, Iran.

4. Department of Nursing, School of Nursing and Midwifery, Arak University of Medical Sciences, Arak, Iran.

5. Department of Epidemiology and Biostatistics, School of Public Health, Tehran University of Medical Sciences, Tehran, Iran.

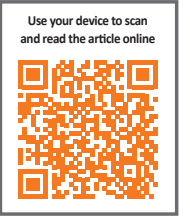

Cftration: Naseri Salahshour, V., et al., 2017. The Effect of Body Position and Early Ambulation on Comfort, Bleeding, and Ecchymosis After Diagnostic Cardiac Catheterization. Journal of Client-Centered Nursing Care, 3(1), pp. 19-26. https://doi. org/10.32598/jccnc.3.1.19

: https://doi.org/10.32598/jccnc.3.1.19

Article info:

Received: 16 Sep. 2016 Accepted: 20 Dec. 2016

\begin{abstract}
A B S T RA C T
Background: Diagnostic cardiac catheterization is a common procedure in coronary artery disease Patients' movement and activities are limited because of potential risks; therefore, they need bed rest. The current study aimed at investigating the impacts of changes in body position and earlier ambulation on comfort, bleeding, and ecchymosis after the diagnostic cardiac catheterization.

Methods: The current clinical trial with quasi-experimental design included 90 patients undergoing diagnostic catheterization hospitalized in Shariati Hospital, Tehran, Iran. The purposive sampling method was used in the current study and subjects were allocated into 2 groups of intervention and control each of 45 . The control group received the routine practices, the supine and flat rest for 8 to 24 hours, and sandbag for 8 hours. The intervention group changed their position in bed; first hour in flat position and head of bed in $15^{\circ}$ and second hour in flat position and head of bed in $30^{\circ}$; then, in the $3^{\text {rd }}$ hour in $45^{\circ}$ position as well as applying sandbag on catheter entrance site for the first 3 hours; then, the patient was allowed to rest in any position $\left(15^{\circ}\right.$ to $\left.30^{\circ}\right)$. Levels of comfort as well as the amount of bleeding and ecchymosis were studied immediately after the admission, 6 hours, 24 hours, and 7 days after the catheterization. Then, the results were analyzed using Chi-square and the Mann-Whitney tests with SPSS V. 11.5.
\end{abstract}

Results: There was no significant difference in the levels of comfort as well as the amount of bleeding and ecchymosis in early admission between the 2 groups, but the level of comfort was higher in the intervention group than the control group at the hours 6 and 24 after the catheterization ( $\mathrm{P}<0.001)$; in addition, there were no statically significant difference between the intervention and control groups in the amount of bleeding and ecchymosis at 6 hours, 24 hours, and 7 days after the catheterization $(\mathrm{P}=0.99)$.

Conclusion: It was observed that patients may be allowed to change their bed position, and they may be ambulated earlier (the sixth hour) after the diagnostic cardiac catheterization.

\section{* Corresponding Author:}

Masoume Sabzali Gol, MSc.

Address: Department of Medical-Surgical Nursing, Faculty of Nursing and Midwifery, Karaj Branch, Islamic Azad University, Karaj, Iran. Tel: +98 (25) 52343395

E-mail:msabzaligol@gmail.com 


\section{Background}

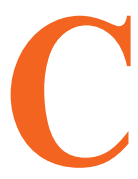

ardiovascular diseases are the most common serious illness in the developed countries and the first cause of death in males and females of all races and ages (Alters \& Schiff 2009). Coronary artery diseases are the most common chronic and life threatening illness among cardiovascular diseases, which bring about disability, economic damage, and death more than any other illness (Bahr 2000), and involve $4 \%$ to $6 \%$ of people in any country (Hashemy \& Zakerimoghadam 2013; Torabi, Salavati \& Sarabi 2012). Cardiovascular diseases are the second common cause of death for both genders in Iran (Sardarinia et al. 2016). Fortunately, new methods of cardiovascular diagnosis and treatment are successful to decrease $50 \%$ of mortality rate, among which cardiac catheterization and angiography are really valuable. Coronary angiography is a selective method to confirm or reject coronary artery diseases to gather necessary data to decide on medication, angioplasty, or Coronary Artery Bypass Graft (CABG) surgery (Hinkle \& Cheever 2013; Nettina, 2013; Foji et al. 2014) and is used as an important diagnostic method for coronary artery disease, renal arteries, abdominal mesenteric arteries, and carotid arteries (Kato et al. 2009).

Different studies show that it is very common to develop some complications such as hemorrhage, hematoma, embolism, arterial fistula to the vein, abscess, arterial aneurysm, and ecchymosis in distal area from catheter entrance due to great vessel trauma (Bakhshi et al. 2014; Castillo-Sang et al. 2010), among which hemorrhage is the most common one (Dabbs et al. 1999). To prevent such complications, specifically bleeding at the catheter entrance site, depending on the routines of the place, the patient is put to 4 to 24 hours flat position bed rest (Bakhshi et al. 2014; Mohammady et al. 2014). That is why patients mostly complain about their movement limitation and long-term immobilization in the bed (Lundén, Bengtson \& Lundgren 2006).

Symptoms associated with bleeding and hematoma normally appear after 12 hours, which may result in blood pressure drop or decreased hematocrit (Dubey \& Sharma 2012). Long-term immobilization and flat rest in bed result in patient's discomfort including difficulties in eating, communicating as well as urinating and defecating (Layman 1996). There may appear some negative psychological impacts due to difficulties in communicating with others in addition to physical discomfort, which adds to the nurses' workload to give more services (Dabbs et al. 1999). Studies show that decreasing flat bed rest-time and early ambulation significantly help to reduce pain and give comfort after catheterization without increasing the risk of bleeding at the catheter entrance site (Rosenstein et al. 2004; Tengiz et al. 2003). Studies also showed that decreasing bed rest to 90 minutes (Gall et al. 2006) and even an hour (Doyle et al. 2006) does not significantly increase the risk of bleeding and hematoma; therefore, it can be considered as a safe and affordable method. It is repeatedly observed that the patients complain about back pain due to long-term flat bed rest, in most of which the nurse's help to convince the patient is of no use (Rezaei-Adaryani 2006).

The current study aimed at defining the impact of body position and early ambulation on patient's comfort, hemorrhage, and ecchymosis, after cardiac catheterization.

\section{Materials \& Methods}

\section{Study type}

The current clinical trial with quasi-experimental design was conducted in Shariati Hospital, Tehran, Iran.

\section{Ethical considerations}

The protocol of the current study was approved by the Ethics Committee of Tehran University of Medical Sciences, Tehran, Iran, under the code 250/4817. Patients participating in the current study signed the written consent.

\section{Sampling}

A total of 90 patients hospitalized for catheterization enrolled in the current study. To define the exact sample size, a pilot study was conducted on 22 patients, and accordingly, the levels of comfort in entering the ward and after 6 hours of intervention were measured. The difference between the mean (SD) of comfort in the two levels was $1.77 \pm 3.19$, with $\beta=0.05$ and $\alpha=0.05$. Therefore, the sample size was calculated as 42 in each group, which changed to 45 to predict the study dropouts.

The eligible patients who met the inclusion criteria were included. The inclusion criteria were: age range 18 to 65 years, Body Mass Index (BMI) of 19 to 23 $\mathrm{kg} / \mathrm{m}^{2}$, drainage fraction $>40.0 \%$, systolic blood pressure $<180 \mathrm{mmHg}$ and diastolic blood pressure $<100$ $\mathrm{mmHg}$, complete consciousness, lack of active bleeding, peripheral vascular coagulation diseases, diabetes, and sensory-motor problems, Partial Thromboplastin Time $(\mathrm{PTT})<90$ seconds, Prothrombin Time $(\mathrm{PT})<16$ seconds, no use of anticoagulant drugs before catheter- 
ization, lack of emergency catheterization, and femoral artery catheterization using 4 to 6 French catheters, no vein or artery tear during angiography, and no significant complications such as hemodynamic changes, bleeding, and dangerous rhythm disorder immediately after the catheterization. Exclusion criteria were: emergence of hemodynamic impairment during the study (reduced systolic or diastolic blood pressure by $20 \mathrm{~mm} \mathrm{Hg}$; pulse rate increased or decreased by more than 20 beats per minute; respiration rate $>35$ or $<10$ breaths per minute), and also feeling general discomfort.

Since there were two groups of intervention and control in the current study, subjects of the intervention group were selected by tossing and then, rest of the participants were assigned to the control group.

\section{Data collection}

Interviews, observations, and self-reports were used to collect data in the current study. The tools consisted of demographic questionnaire, comfort checklist, bleeding checklist, and measurement of ecchymosis. First, the demographic questionnaire was completed, and then, the study started after doing the dressing. The comfort checklist was filled out to examine the level of comfort during the intervention in 3 periods (entering the ward, 6 hours, and 24 hours after starting the study) by interviews. Categorization was made at four levels: discomfort (0), low comfort (1 to 3), medium comfort (4 to 8), and high comfort (9 to 10). The checklist consisted of questions about the level of patient's comfort in eating, drinking, urinating, etc.

The bleeding checklist was developed in 4 time periods ( 3 as mentioned above and 7 days after starting the study), which was completed based on the observations. The bleeding was categorized in 3 levels: no bleeding, minor bleeding (no hemodynamic impairment and no need for blood transfusion), and major bleeding (with hemodynamic impairment and need for blood transfusion). The tool to measure ecchymosis consisted of a long axis and a transversal axis, each graded in centimeters to measure the area of ecchymosis by observation in 4 time periods and to categorize in 5 levels: no ecchymosis, small ecchymosis ( 1 to $40 \mathrm{~cm}^{2}$ ), medium ecchymosis (41 to $80 \mathrm{~cm}^{2}$ ), large ecchymosis ( 81 to $100 \mathrm{~cm}^{2}$ ), and very large ecchymosis $\left(>100 \mathrm{~cm}^{2}\right)$.

The tools used in the current study were made based on the related books and articles, and the professional experiences of nursing professors and university cardiologists at Tehran University of Medical Sciences. Content vali- dation was used to determine the scientific credibility of the tools. It means that some tools were developed after carefully studying the books and references on the subject matter, and then, they were given to the 10 faculty members in Midwifery Department at Tehran University of Medical Sciences to comment on them. Accordingly, they were used in the study following the modifications. Scientific validity of the tool to measure bleeding and ecchymosis was reported 0.80 by Cronbach's alpha, and 0.92 to 0.98 by the test retest method, which indicated the reliability of the tool. Cronbach's alpha $(\alpha=0.80)$ was used to determine the validity of the comfort checklist.

The level of comfort, ecchymosis, and bleeding were measured and recorded by the developed tools. In addition, the level of complications were measured and recorded for both groups after 24 hours. The amount of bleeding and ecchymosis were measured and recorded after 7 days (the level of comfort was not measured due to the impact of different factors). Patients usually refer to the catheterization ward after a week to receive the test results, and then, they are referred to the clinic to be visited by a specialist. The researcher was ready in both places on the day 7 to investigate the bleeding complication and record the observations. But, the candidates for CABG were referred to the cardiac surgery department one day after catheterization. There, they were either placed in the waiting list for a week or underwent the cardiac surgery and, the researcher investigated them accordingly (23 patients experienced such conditions).

\section{Intervention}

The patients in the intervention group were placed in bed since admission for one hour in flat position while the head of bed in $15^{\circ}$ and in the second hour in flat position and head of bed in $30^{\circ}$, then in the third hour in $45^{\circ}$ position; for the first 3 hours a $2 \mathrm{~kg}$ sandbag placed on the catheter placement site and after 3 hours, patients were allowed to rest in any position $\left(15^{\circ}\right.$ to $\left.45^{\circ}\right)$. While changing the position, the patients pressed the pressure dressing put by the researcher on the placement site of catheter by the help of fingers (taught previously) and the sandbag. If there were any bleeding, hematoma, or ecchymosis during these 6 hours, the patient would immediately be put in $15^{\circ}$ and flat position to get predictive cares for the complications.

It is noteworthy that in such cases the intervention procedure was stopped and the exclusion criteria were recorded and reported. At the end of the hour 6, study variables (level of comfort as well as the amount of ecchymosis and bleeding) were investigated in both 
groups. Then, the patients in the intervention group slowly departed the bed; at first sat 5 minutes with hanging feet, and then, walked with the help of the researcher. All complications were carefully observed during ambulation and walking in the ward. If there were any complication, the patient was immediately returned to the bed for necessary practices. In the control group, all routine post-catheterization cares were given (The patient was placed in flat position for 8 to 24 hours in $0^{\circ}$ with sandbag on the catheterization site for 8 hours).

\section{Data analysis}

Data was analyzed by descriptive statistics and the Kolmogorov-Smirnov, Chi-square, and Mann-Whitney tests with SPSS V. 11.5. P-value less than 0.05 was considered as significant.

\section{Results}

A patient from the control group was excluded from the study due to receiving a dose of heparin based on the physician's prescription for double vision after catheterization, as it would affect the amount of bleeding and ecchymosis. No patient from the intervention group was excluded from the study following the complications such as bleeding, hematoma, and/or ecchymosis. Therefore, there were 44 patients in the control group and 45 in the intervention group.
Both groups were homogeneous in term of the demographic data (Table 1). The current study findings showed no significant difference between the groups regarding the level of comfort at the time of admission; hence, both groups were homogeneous from the first stage. The level of comfort after 6 hours was $86.7 \%$ and $100 \%$ for the control and intervention groups, respectively. There was a significant difference between the two groups regarding the comfort after 6 hours, according to the MannWhitney test $(\mathrm{P}<0.001)$. Mean of comfort was higher in the intervention group, compared with that of the control group after 6 hours (Table 2). There was also a significant difference in the comfort level 24 hours after the study onset $(\mathrm{P}<0.001)$.

Comparison of the means showed that comfort level was higher in the intervention group (Table 2). Most of the subjects studied in both intervention and control groups had no bleeding at the time of admission, and there was clearly no significant difference in this regard between the groups and the groups were homogenous. No significant difference was observed between the groups in the amount of bleeding after 6 hours. It was also clarified that there was no significant difference between the groups in the amount of bleeding after 24 hours. In the seventh day follow-up, none of the subjects had bleeding, and there was no significant difference between the groups (Table 3).

Table 1. Demographic data of the study groups $(n=90)$

\begin{tabular}{|c|c|c|c|c|}
\hline \multirow{2}{*}{ Variable } & \multirow{2}{*}{ Group } & Control & Intervention & \multirow{2}{*}{$\mathbf{P}$} \\
\hline & & No. (\%) & No. (\%) & \\
\hline \multirow{5}{*}{ Age (year) } & $18-35$ & $3(6.6)$ & $4(9.1)$ & \multirow{5}{*}{0.61} \\
\hline & $36-45$ & $5(11.1)$ & $6(13.6)$ & \\
\hline & & & & \\
\hline & $46-55$ & $17(37.7)$ & $18(40.9)$ & \\
\hline & $56-65$ & $20(44.4)$ & $16(36.4)$ & \\
\hline \multirow{3}{*}{ Gender } & Male & $19(42.2)$ & $25(56.8)$ & \multirow{3}{*}{0.20} \\
\hline & & & & \\
\hline & Female & $26(57.7)$ & $19(43.2)$ & \\
\hline \multirow{5}{*}{ Level of education } & Illiterate & $6(13.3)$ & $4(9.1)$ & \multirow{5}{*}{0.94} \\
\hline & Primary school & $6(13.3)$ & $4(9.1)$ & \\
\hline & Secondary school & $13(28.2)$ & $13(29.5)$ & \\
\hline & High school & $11(24.4)$ & $12(27.3)$ & \\
\hline & Higher education & $2(4.4)$ & $3(6.8)$ & \\
\hline
\end{tabular}


Table 2. Comparison of the level of comfort in patients who underwent cardiac diagnostic catheterization, based on the followup time and study group $(\mathrm{n}=90)$

\begin{tabular}{|c|c|c|c|c|}
\hline \multirow{2}{*}{$\begin{array}{c}\text { Time } \\
\text { Group }\end{array}$} & \multicolumn{2}{|c|}{6 Hours After Angiography } & \multicolumn{2}{|c|}{24 Hours After Angiography } \\
\hline & Control & Intervention & Control & Intervention \\
\hline Comfort & No. (\%) & No. (\%) & No. (\%) & No. (\%) \\
\hline Medium & $39(86.7)$ & 0.0 & $45(100.0)$ & $44(100.0)$ \\
\hline High & $6(13.3)$ & $44(100.0)$ & $45(100.0)$ & $44(100.0)$ \\
\hline Mean (SD) & $0.208(6.96)$ & $1.11(7.36)$ & $9.64(0.48)$ & $10(0.00)$ \\
\hline Mann-Whitney Test & \multicolumn{2}{|c|}{$\begin{array}{l}Z=-8.65 \\
P<0.001\end{array}$} & \multicolumn{2}{|c|}{$\begin{array}{l}Z=-4.38 \\
P<0.001\end{array}$} \\
\hline
\end{tabular}

SD: Standard Deviation

Client-Centered Nursing Care

Table 3. Comparison of the amount of bleeding in patients who underwent cardiac diagnostic catheterization, based on the follow-up time and study group $(\mathrm{n}=90)$

\begin{tabular}{|c|c|c|c|c|c|c|}
\hline \multirow{2}{*}{$\begin{array}{l}\text { Time } \\
\text { Group }\end{array}$} & \multicolumn{2}{|c|}{6 Hours After Angiography } & \multicolumn{2}{|c|}{24 Hours After Angiography } & \multicolumn{2}{|c|}{7 Days After Angiography } \\
\hline & Control & Intervention & Control & Intervention & Control & Intervention \\
\hline Bleeding & No. (\%) & No. (\%) & No. (\%) & No. (\%) & No. (\%) & No. (\%) \\
\hline No bleeding & $45(100.0)$ & $44(100.0)$ & $45(100.0)$ & $44(100.0)$ & $45(100.0)$ & $44(100.0)$ \\
\hline Mean (SD) & $10(0.00)$ & $10(0.00)$ & $10(0.00)$ & $10(0.00)$ & $10(0.00)$ & $10(0.00)$ \\
\hline Mann-Whitney Test & \multicolumn{2}{|c|}{$\begin{array}{c}Z=0.00 \\
P=1\end{array}$} & \multicolumn{2}{|c|}{$\begin{array}{c}Z=0.00 \\
P=1\end{array}$} & \multicolumn{2}{|c|}{$\begin{array}{c}Z=0.00 \\
P=1\end{array}$} \\
\hline
\end{tabular}

The amount of ecchymosis was investigated at the time of admission and there was no ecchymosis in most of the subjects in both groups. The current study findings indicated no significant difference between the groups in ecchymosis and they were homogenous at the beginning. There was clearly no significant difference in ecchymosis after 6 hours (Table 4). The current study re- sults showed neither significant difference in ecchymosis after 24 hours nor the seventh day follow-up.

\section{Discussion}

The average rate of comfort for the study subjects 6 hours after enrollment in the study was higher in the in-

Table 4. Comparison of the amount of ecchymosis in patients who underwent cardiac diagnostic catheterization, based on the follow-up time and study group $(n=90)$

\begin{tabular}{|c|c|c|c|c|c|c|}
\hline \multirow{2}{*}{$\begin{array}{c}\text { Time } \\
\text { Group }\end{array}$} & \multicolumn{2}{|c|}{6 Hours After Angiography } & \multicolumn{2}{|c|}{24 hours After Angiography } & \multicolumn{2}{|c|}{7 Days After Angiography } \\
\hline & Control & Intervention & Control & Intervention & Control & Intervention \\
\hline Comfort & No. (\%) & No. (\%) & No. (\%) & No. (\%) & No. (\%) & No. (\%) \\
\hline No ecchymosis & $28(62.2)$ & $25(56.8)$ & $28(62.2)$ & $23(52.3)$ & $8(17.7)$ & $9(20.4)$ \\
\hline Small ecchymosis & $15(33.4)$ & $19(43.2)$ & $15(33.4)$ & $21(47.7)$ & $18(40.0)$ & $18(40.9)$ \\
\hline $\begin{array}{l}\text { Medium ecchy- } \\
\text { mosis }\end{array}$ & $2(4.4)$ & 0.0 & $2(4.4)$ & 0.0 & $6(13.3)$ & $6(13.6)$ \\
\hline Mean (SD) & $\begin{array}{l}13.01 \\
(4.69)\end{array}$ & $6.38(3.49)$ & $13.01(4.71)$ & $6.48(3.89)$ & $3.01(4.69)$ & $3.01(4.69)$ \\
\hline Mann-Whitney Test & \multicolumn{2}{|c|}{$\begin{array}{l}Z=-0.68 \\
P=0.49\end{array}$} & \multicolumn{2}{|c|}{$\begin{array}{l}Z=-0.77 \\
P=0.43\end{array}$} & \multicolumn{2}{|c|}{$\begin{aligned} Z & =-0.46 \\
P & =0.64\end{aligned}$} \\
\hline
\end{tabular}


tervention group than the control group. The difference seemed related to the change in the patients' positions and in ambulation 6 hours after catheterization, which was at least 8 to 12 hours for the control group to stay in flat position.

It seems that ambulation helps the patient to have better capabilities in eating, drinking, urinating and defecating as well as communicating with the nurses to meet the personal and primary needs. The results also showed that the level of comfort after 24 hours in the control group was less than that of the intervention group. Since the patients in the control group left the bed after 8 to 12 hours, the level of comfort increased in them accordingly. According to a study entitled "Early Ambulation After Diagnostic Angiography Using 4-f Catheters and Sheaths", there was no significant difference 24 hours after the catheterization between the control and intervention groups regarding the level of comfort (Dowling et al. 2002). Similarity of the amount of comfort in both groups may result from the ambulation of the patients in the control group 6 hours after the catheterization and in the intervention group after 3 hours that made normal level of comfort after 24 hours.

One of the most important objectives of putting patients in flat position is to prevent the catheterization site bleeding; yet in current study no significant difference was observed between the two groups regarding the amount of bleeding, despite different positions the patients had on the beds and that the patients in the intervention group left their beds (after 6 hours) earlier than the subjects in the control group. In the study by Yilmaz et al., entitled "Minimizing Short-term Complications in Patients Underwent Cardiac Invasive Procedure" on 169 candidates for the cardiac catheterization, the first group received the application of $4.5 \mathrm{~kg}$ sandbags and the second group $2.3 \mathrm{~kg}$ sandbags for two hours on the catheter entrance site. Yet the results showed no difference in the amount of bleeding between the groups (Yilmaz, Gurgun \& Dramali 2007).

In a study by Bakhshi et al., entitled "Effect of Positioning on Patient Outcomes After Coronary Angiography" on 80 candidates of non-emergency cardiac catheterization in Yazd, Iran, the patients were randomly allocated into two groups of intervention and control; there was no significant difference in bleeding after two hours between the groups. In that study, in addition to routine medical care practices, the intervention group left the bed with no limitation 6 hours after the catheterization. The average amount of ecchymosis after 6 hours was a little higher in the control group, but not significantly (Bakhshi et al. 2014).
In other words, though the goal of immobilizing and long-term bed rest was to prevent the complications such as bleeding and ecchymosis, there were some patients who developed ecchymosis even in the control group. It might be just a random case; therefore, it is necessary to have other studies with more samples to make sure about the results, yet it seems that changing the patient's position and patient's early ambulation do not increase the risk of ecchymosis. In the study by Yilmaz et al., no significant difference was observed between the groups regarding the amount of ecchymosis.

The current study results indicated no significant difference in the amount of bleeding between the groups 24 hours after the catheterization. In other words, no bleeding was observed in the study subjects after 24 hours. It can be said that patient's early ambulating had no impact on bleeding and clotting from the catheter site. The same results were observed in similar studies by other researchers. The study by Yilmaz et al. showed no significant difference in bleeding one day after angiography (Rezaei-Adaryani 2006). The average amount of ecchymosis was a little higher in the control group 24 hours after the catheterization.

The Mann-Whitney U-test showed no significant difference at the $24^{\text {th }}$ hour. It indicated that patients' change of position and early ambulation had no impact on developing ecchymosis, and that little difference might be attributed to a little higher ecchymosis of the intervention group from the beginning. The study by Yilmaz et al. showed no significant difference in the ecchymosis one day after angiography (Rezaei-Adaryani 2006). The analysis of the findings revealed no significant difference in the amount of bleeding on the seventh day. In other words, no one had bleeding on the day 7 . This may clarify that patients' change of position and early ambulating caused no bleeding even after a longer time. In the study by Dowling et al. there was no significant difference even after 30 days in bleeding between the two groups (Yilmaz, Gurgun \& Dramali 2007), which indicated that even in longer follow-ups patients showed no difference.

In other words, patients' early ambulation and change of position had no effects on bleeding after 24 hours and ever later. The average amount of ecchymosis on the seventh day was a little higher in the intervention group, but the difference was insignificant. Though patients normally develop ecchymosis after catheterization, and this complication increases during the time, patients' change of position and early ambulation caused no development of ecchymosis, compared with that of the control group. Therefore, it seems that the intervention had the same impact on pa- 
tients' ecchymosis development, compared to that of the patients in the control group, and early ambulation and movements made no difference more than expected.

Post-catheterization cares given to some of the patient, removal of the artery sheath by different nurses, the impact of pressure imposed to create homeostasis on some complications such as ecchymosis, hematoma, and bleeding were the factors that could not be controlled by the authors. Since different methods are used in hospital ward to create homeostasis, further studies on the other hemostasis management methods, such as the manual ones, using the same methodology is recommended.

The results indicated that it was possible to safely change the position of the patients after diagnostic cardiac catheterization and ambulate them earlier (the sixth hour). The results suggested a method to increase the patients' comfort and improve the nurses' knowledge as the main element of patient care to enhance the quality of nursing care after the diagnostic catheterization or cardiac treatment. In addition, patients' early ambulation after catheterization can reduce the time of hospitalization and accordingly the associated complications.

\section{Acknowledgements}

This paper is extracted from the MSc. thesis of the first author with the financial support of Research Deputy of Tehran University of Medical Sciences and Health Services. The authors are thankful to Research Deputy of Tehran University of Medical Sciences and Health Services, Research Deputy of Faculty of Nursing and Midwifery, professors at Faculty of Nursing and Midwifery as well as all physicians, nurses, and patients at Shariati Hospital, Tehran, Iran, who kindly helped with the study.

\section{Conflict of Interest}

The authors declared no conflicts of interest.

\section{References}

Alters, S. \& Schiff, W., 2009. Essential concepts for healthy living. Massachusetts: Jones \& Bartlett Publishers.

Bahr, R. D., 2000. The early heart attack care program, catheterization. Journal of Cardiovascular Diseases \& Diagnosis, 53(3), p. 157.

Bakhshi, F., et al., 2014. Effect of positioning on patient outcomes after coronary angiography. Journal of Nursing Research, 22(1), pp. 45-50. doi: 10.1097/jnr.0000000000000020.
Castillo-Sang, M., et al., 2010. Femoral artery complications after cardiac catheterization: A study of patient profile. Annals of Vascular Surgery, 24(3), pp. 328-35. doi: 10.1016/j. avsg.2009.06.025

Dabbs, A. D. V., et al., 1999. Nursing assessment of patient readiness for ambulation after cardiac catheterization. Medsurg Nursing, 8(5), p. 309. PMID: 10661169

Dowling, K., et al., 2002. Early ambulation after diagnostic angiography using 4-f catheters and sheaths: A feasibility study. Journal of Endovascular Therapy, 9(5), pp. 618-21. doi: 10.1583/1545-1550(2002)009 < 0618:eaadau > 2.0.co;2

Doyle, B. J., et al., 2006. Ambulation 1 hour after diagnostic cardiac catheterization: A prospective study of 1009 procedures. Mayo Clinic Proceedings, 81(12), pp. 1537-40. doi: 10.4065/81.12.1537.

Dubey, L. \& Sharma, S. K., 2012. Cardiac catheterization and complications: initial experience. Journal of College of Medical Sciences-Nepal, 8(2), pp.1-6.

Foji, S., et al., 2015. The study of the effect of guided imagery on pain, anxiety and some other hemodynamic factors in patients undergoing coronary angiography. Complementary Therapies in Clinical Practice, 21(2), pp. 119-23.

Gall, S., et al., 2006. Rapid ambulation after coronary angiography via femoral artery access: A prospective study of 1,000 patients. Journal of Invasive Cardiology, 18(3), pp. 106-8.

Hashemy, S. \& Zakerimoghadam, M., 2013. Comparative study of the effect of muscle relaxation and music therapy on anxiety level in patients waiting for cardiac catheterization. Iranian Journal of Cardiovascular Nursing, 1(4), pp. 22-30.

Hinkle, J. L. \& Cheever, K. H., 2013. Brunner and suddarth's textbook of medical-surgical nursing. Pennsylvania: Lippincott Williams \& Wilkins.

Kato, F., et al., 2009. Reduction of bed rest time after transfemoral noncardiac angiography from 4 hours to 2 hours: A randomized trial and a one-arm study. Journal of Vascular and Interventional Radiology, 20(5), pp. 587-92. doi: 10.1016/j. jvir.2009.02.003.

Layman, T. E., 1996. Reducing time in bed after cardiac catheterization (TIBS II). American Journal of Critical Care: An Official Publication, American Association of Critical-Care Nurses, 5(6), pp. 462. PMID: 8811150

Lundén, M. H., Bengtson, A. \& Lundgren, S. M., 2006 Hours during and after coronary intervention and angiography. Clinical Nursing Research, 15(4), pp. 274-89. doi $10.1177 / 1054773806291855$.

Mohammady, M. et al., 2014. Early ambulation after diagnostic transfemoral catheterisation: A systematic review and metaanalysis. International Journal of Nursing Studies, 51(1), pp. 3950. doi: 10.1016/j.jinurstu.2012.12.018

Nettina, S. M., 2013. Lippincott Manual of Nursing Practice. Pennsylvania: Lippincott Williams \& Wilkins.

Rezaei-Adaryani, M., 2006. [The effect of position on back pain and bleeding after angiogeraphy (Persian)] (PhD dissertation). Tehran: Tarbiat Modares University.

Rosenstein, G., et al., 2004. Simple clinical risk stratification and the safety of ambulation two hours after 6 French diagnostic 
heart catheterization. The Journal of Invasive Cardiology, 16(3), pp. 126-8. PMID: 15152161

Sardarinia, M., et al., 2016. Risk factors for incidence of cardiovascular diseases and all-cause mortality in a middle eastern population over a decade follow-up: Tehran lipid and glucose study. PLOS ONE, 11(12), 0167623. doi: 10.1371/journal. pone. 0167623

Tengiz, I., et al., 2003. Six hour ambulation after elective coronary angioplasty and stenting with 7F guiding catheters ald low dose heparin. Kardiologia Polska, 58(2), pp. 93-7. PubMed ID: 14504634

Torabi, M., Salavati, M. \& Sarabi, A. G., 2012. [Effect of foot reflexology massage and benson relaxation techniques on anxiety and physiological indexes of patients undergoing coronary heart angiography (Persian)]. Scientific Journal of Hamadan Nursing \& Midwifery Faculty, 20(1), pp. 63-73.

Yilmaz, E., Gurgun, C. \& Dramali, A., 2007. Minimizing shortterm complications in patients who have undergone cardiac invasive procedure: A randomized controlled trial involving position change and sandbag. The Anatolian Journal of Cardiology, 7(4), pp. 390-7.

Zakeri-moghadam, M. \& Hashemi, S. 2014. [Investigating the effect of Benson muscle relaxation on cardiac hemodynamic amounts in patients candidated for cardiac catheterization (Persian)]. Nursing Development in Health, 4(6-7), pp. 5-12 